ORIGINAL ARTICLE

\title{
Recall after procedural sedation in the emergency department
}

\author{
Alex Swann, Jeffrey Williams, Daniel M Fatovich
}

Emerg Med J 2007;24:322-324. doi: 10.1136/emj.2006.040923

See end of article for authors' affiliations

....... are

Correspondence to: Dr A Swann, Department of Anaesthesia, Royal Perth Hospital, Box X2213 GPO Perth WA 6847, Australia; alexswann@doctors.org.uk

Accepted 2 February 2007
Background: Procedural sedation (PS) is common in the emergency department (ED) and ideally patients should have no recall of the procedure.

Aim: To determine the incidence of recall.

Methods: A prospective observational study in an university ED of all patients undergoing PS. Data were collected on a pre-formatted data sheet. Levels of satisfaction with the sedation by the treating physician and nurse were recorded on a $10 \mathrm{~cm}$ visual analogue scale. On recovery, the patient was asked a validated questionnaire to determine the rate of immediate recall and at telephone follow-up for delayed recall.

Results: 125 patients (88 male, $70 \%$ ) were enrolled and 110 had completed follow-up. Mean (range) age was 51.6 (13-91) years. Procedures included 84 (67\%) orthopaedic reductions and $41(33 \%)$ cardioversions. A wide range of drug combinations were used, including fentanyl/propofol $32(25.6 \%)$, fentanyl/midazolam 30 (24\%), fentanyl/midazolam/propofol $16(12.8 \%)$, propofol $13(10.4 \%) .87 .2 \%$ of procedures were successful. A grimace/groan was observed in 61 of 125 (49\%). Immediate recall occurred in 9 of 121 (7.4\%; $95 \% \mathrm{Cl} 3.7$ to 14.0) and delayed recall in 5 of 110 (4.5\%; $95 \% \mathrm{Cl} 1.7$ to 10.8$)$. No drug combination was correlated with recall (Spearman's rho $=0.149$ ), nor the presence of a grimace/groan $(r=-0.039)$. Median sedation satisfaction scores were physician 9.0, nurse 10, patient 10. Correlation of delayed recall with patient satisfaction was $-0.471(p<0.001)$.

Conclusions: Recall following PS in ED is uncommon. There is no association of recall with drugs used or the presence of a grimace/groan. There is high patient satisfaction with PS in the ED.
$\mathrm{O}$ ne of the goals of emergency department (ED) procedural sedation (PS) is to induce amnesia for brief painful procedures, in addition to analgaesia. However, few studies have determined the incidence of recall following PS in the emergency department (ED).

In the few studies that examined this issue, the rates of recall ranged from $2.1 \%$ to $35 \%$ for immediate recall, and a $25 \%$ rate of recall at $24 \mathrm{~h}$ after a procedure. ${ }^{1-6}$ These rates are high compared to the $0.1-0.2 \%$ incidence of awareness after general anaesthesia. $^{7}$

In none of the ED studies was recall the primary outcome measure. Therefore, we conducted this study to determine the incidence of recall following PS using current clinical practice. As a secondary end point, we aimed to identify any associated factors.

\section{METHODS}

Study design

This was a prospective observational study from January to August 2005. All patients undergoing PS were eligible for inclusion. Patients were excluded from the study if they had an altered mental state or had an inability to understand the questions (eg, language problems). The project was approved by the hospital's Clinical Safety and Quality Unit and was granted an exemption by the ethics committee.

Patients undergoing procedural sedation had ECG, noninvasive blood pressure and pulse oximetry monitoring with supplemental oxygen. The treating physician was a consultant or registrar in emergency medicine who clinically determined the drugs and doses used.

\section{Study setting and population}

The Royal Perth Hospital, Perth, Western Australia, Australia, is an adult university hospital with an annual census of 53000 and an admission rate of $42 \%$.

\section{Study protocol}

A pre-formatted data sheet was contemporaneously completed, with sections for the treating physician and nurse. The data recorded included age, sex, weight, type of procedure, drugs and doses used, procedural success, complications and if the patient was observed to grimace or groan. The physician performing the sedation was asked to complete a $10 \mathrm{~cm}$ visual analogue scale (VAS) in response to the question: "How satisfied were you with the sedation?" with zero being completely dissatisfied and 10 being completely satisfied. A similar VAS was independently completed by the nurse. Relevant time intervals were recorded.

Once the nurse had clinically determined that the patient had recovered from the procedural sedation, the patient was asked a validated awareness questionnaire ${ }^{8}$ (immediate recall) which included the following questions:

(1) What was the last thing you remembered before the procedure?

(2) What was the next thing you remember?

(3) Can you remember anything in between these two periods?

(4) Can you remember dreaming during your procedure?

(5) If yes, did you notice sounds or conversations, paralysis, touch or movement, visual perceptions or pain.

Patients were requested to give consent for a follow-up telephone call within $72 \mathrm{~h}$ (delayed recall) to answer the following questions:

(1) Using a scale from 0 to 10 with $0=$ none of it and $10=$ all of it, after you were given something to make you sleepy, how much of the procedure do you remember? This technique was reported by Miner. ${ }^{1}$

Abbreviations: $E D$, emergency department; PS, procedural sedation; VAS, visual analogue scale 
Table 1 Drug combinations used

\begin{tabular}{lrr}
\hline Drug combinations & $\mathbf{n}$ & $\%$ \\
\hline Fentanyl-propofol & 32 & 25.6 \\
Fentanyl-midazolam & 30 & 24.0 \\
Fentanyl-midazolam-propofol & 16 & 12.8 \\
Propofol & 13 & 10.4 \\
Midazolam-morphine & 11 & 8.8 \\
Fentanyl-morphine-propofol & 6 & 4.8 \\
Morphine-propofol & 5 & 4.0 \\
Midazolam & 3 & 2.4 \\
Fentanyl-midazolam-morphine & 3 & 2.4 \\
Midazolam-propofol & 3 & 2.4 \\
Midazolam-morphine-propofol & 2 & 1.6 \\
Ketamine & 1 & 0.8 \\
& 125 & 100 \\
\hline
\end{tabular}

(2) Using a scale from 0 to 10 with $0=$ completely dissatisfied and $10=$ completely satisfied, how satisfied were you with the sedation?

(3) Did you experience any untoward effects the next day?

\section{Measurements}

The proportion of patients who experienced immediate and delayed recall.

\section{Data analysis}

As this was only a descriptive study, no power calculation was undertaken. Data were recorded and analysed with SPSS v.12. Standard descriptives were obtained. Statistical comparison was made between groups using Spearman's correlation. Significance was set at $\mathrm{p}=0.05$.

\section{RESULTS}

In all, 125 patients were enrolled, and 15 were lost to follow-up but were still included in the analysis. The mean (range) age was 51.6 (13-91) years, and $88(70.4 \%)$ were male. The mean weight of the patients was $79.6 \mathrm{~kg}$ (range 46-120). In all, 84 $(67.2 \%)$ patients were sedated for an orthopaedic reduction and $41(32.8 \%)$ for a cardioversion. Table 1 describes the drug combinations used. The mean total drug doses used were: fentanyl $1.0(0.2-2.6) \mu \mathrm{g} / \mathrm{kg}$, midazolam $0.06(0.01-0.24) \mathrm{mg} /$ $\mathrm{kg}$, morphine $0.1(0.03-0.3) \mathrm{mg} / \mathrm{kg}$ and propofol $1.3(0.4-$ 3.0) $\mathrm{mg} / \mathrm{kg}$.

Procedural success was achieved for 109 (87.2\%) patients. Only $2(1.6 \%)$ patients had a complication associated with the sedation (mild hypotension, delirium). No episodes of desaturation were recorded. Altogether, 61 (48.8\%) patients were observed to grimace or groan during the procedure.

The mean duration of the procedure was 10.7 (1-60) min and the mean (range) time questions were asked after the end of the procedure was 44.1 (1-356) min. The mean (range) time for telephone follow-up was $132.5 \mathrm{~h}$ (median 75.5, IQR 50.7145.0, range 15-883).

In response to the question: Can you remember anything between these two periods? (ie, between the start of PS and waking), 9 of $121(7.4 \%, 95 \%$ CI $3.7 \%$ to $14.0 \%)$ patients reported remembering something, and $14(11.7 \%)$ reported dreaming during the procedure.

At telephone follow-up, 5 of $110(4.5 \%, 95 \%$ CI $1.7 \%$ to $10.8 \%$ ) patients reported remembering something (mean 3.6/ $10)$ of the procedure. This was most commonly described as some memory of pulling and talking for dislocations, and memory of the shock and pain for cardioversion. Dreaming was common. In all, 94 of 108 patients (87\%) reported no untoward effects the next day. Of the 14 that did, symptoms reported included: 6 (43\%) hangover-drowsy, 5 (36\%) nausea, 2 vivid dreams and 1 headache.

The median (range) satisfaction scores were: physician 9.0 $(2-10)$, nurse $10(2.8-10)$ and patient $10(3-10)$. In all, 99 patient scores were 10 and of the remainder, only one scored $<8$. Table 2 reports the correlations of various parameters.

\section{DISCUSSION}

This study shows that a wide variety of drug combinations are used for PS in the ED. We found a low incidence of recall, regardless of the drug combination used. No factor was identified that was associated with immediate recall, and the only factor associated with delayed recall was the physician's satisfaction with the sedation-that is, the less satisfied the physician, the more likely there was delayed recall. There are no obvious reasons for this finding. This delayed recall was associated with a less satisfied patient.

Physicians, nurses and patients were highly satisfied with the sedation. Physicians consistently scored themselves lower than nurses and patients. The main associated factor was that if the patient grimaced or groaned during the procedure, then physician and nurse satisfaction with the sedation was decreased. (About half our patients were observed to grimace or groan, and this is consistent with previous reports.) $)^{4}$ This is probably a subjective judgement that the patient is experiencing pain during the procedure. However, this had no effect on patient satisfaction, the likelihood of procedural success or the presence of any recall. This should be reassuring for clinical staff. Our procedural success rate of $87 \%$ is consistent with previous reports. ${ }^{9}$

The use of any propofol for the PS was associated with higher satisfaction scores for physicians, nurses and patients and no

Table 2 Cross tabulations comparing various parameters

\begin{tabular}{|c|c|c|}
\hline & Spearman's rho & p Value \\
\hline \multicolumn{3}{|l|}{ Correlation of grimace/groan with } \\
\hline Drug combination & 0.076 & 0.398 \\
\hline Procedural success & 0.057 & 0.527 \\
\hline Physician satisfaction & -0.469 & $<0.001$ \\
\hline Nurse satisfaction & -0.323 & $<0.001$ \\
\hline Patient satisfaction & 0.118 & 0.221 \\
\hline Immediate recall & 0.043 & 0.638 \\
\hline Delayed recall & -0.039 & 0.687 \\
\hline \multicolumn{3}{|c|}{ Correlation of immediate recall with } \\
\hline Drug combination & 0.059 & 0.522 \\
\hline Procedural success & -0.018 & 0.847 \\
\hline Doctor satisfaction & -0.128 & 0.169 \\
\hline Nurse satisfaction & 0.029 & 0.748 \\
\hline Patient satisfaction & -0.103 & 0.282 \\
\hline Untoward effects the next day & 0.068 & 0.483 \\
\hline \multicolumn{3}{|l|}{ Correlation of delayed recall with } \\
\hline Drug combination & 0.149 & 0.122 \\
\hline Procedural success & 0.032 & 0.744 \\
\hline Physician satisfaction & -0.196 & 0.04 \\
\hline Nurse satisfaction & -0.127 & 0.188 \\
\hline Patient satisfaction & -0.471 & $<0.001$ \\
\hline Untoward effects the next day & 0.173 & 0.074 \\
\hline \multicolumn{3}{|l|}{ Correlation of propofol use with } \\
\hline Physician satisfaction & 0.281 & 0.002 \\
\hline Nurse satisfaction & 0.278 & 0.002 \\
\hline Patient satisfaction & 0.227 & 0.017 \\
\hline Immediate recall & -0.038 & 0.683 \\
\hline Delayed recall & -0.089 & 0.357 \\
\hline Untoward effects the next day & -0.100 & 0.304 \\
\hline \multicolumn{3}{|l|}{$\begin{array}{l}\text { Correlation of untoward effects the } \\
\text { next day with }\end{array}$} \\
\hline $\begin{array}{l}\text { Drug combination } \\
\text { Correlation of midazolam use with }\end{array}$ & 0.225 & 0.019 \\
\hline Immediate recall & -0.015 & 0.869 \\
\hline Delayed recall & -0.207 & 0.03 \\
\hline Patient satisfaction & 0.218 & 0.022 \\
\hline
\end{tabular}


untoward effects the next day. However, the use of midazolam was associated with a decreased likelihood of delayed recall and a higher patient satisfaction. This is consistent with studies which show that Midazolam causes anterograde amnesia in a dose-responsive manner. ${ }^{10}$ Nevertheless, our study was underpowered to discuss these questions and these results are only hypothesis generating.

Our results highlight the importance of delayed recall as a factor in patient satisfaction, something that is not often considered. We had believed that the drug regimen used was likely to be a major factor. However, we found no correlation of recall with the drug combination used, except that delayed recall was less likely with midazolam.

The range of recall rates reported may reflect both the drugs and measurement tools used. ${ }^{246}$ Lerman et al ${ }^{3}$ reported a prospective observational study of 76 adult patients, to evaluate the use of intravenous methohexital. Using a Likert scale of 15, patients reported little recall of the procedure (mean (SD) 1.3 (0.9)). Wright et al reported 69 patients who received midazolam or diazepam for PS. Using a $10 \mathrm{~cm}$ VAS, patients receiving midazolam (mean (SD) 4.8 (3.6)) had significantly less recall than those in the diazepam group $(6.9$ (2.8)) for the procedure $(\mathrm{p}<0.02)$. However, Dunn et al ${ }^{11}$ reported that 10 of 11 patients had recall, including 9 with full recall, when given a combination of propofol and remifentanil for the reduction of shoulder dislocations

The features of the patients who experienced both immediate and delayed recall in our study do not convey any obvious pattern. There is an impression that some patients' drug doses were "light" for their weight, but this was not consistent. All these patients had only limited delayed recall of the procedure. It is noteworthy that some patients with recall had perfect satisfaction scores from all three scorers, but still had recall. More research is needed to investigate this, as it is likely to be a complex multidimensional problem.

\section{LIMITATIONS}

The main limitation was the wide variety of drug combinations used. Hence, subgroup analysis of drug combinations with recall was limited. However, this variety does reflect clinical practice. Further research in this area would need to use predetermined drug regimens. In addition to this, the time of asking questions after a procedure was not standardised, as reflected in the extremely wide variation in recorded times. This probably relates to working in a busy ED with competing clinical priorities.

Another limitation is that there was no objective measure of the depth of sedation recorded. This could be highly relevant to the issue of recall. One possible solution would be to use bispectral index monitoring. Although this has been studied for PS in the ED, the poor correlation with sedation scores is problematic. ${ }^{12}{ }^{13}$ A range of sedation scores are available for use. However, this was a clinical study whereby the treating clinical staff titrated their PS to a clinical end point and not to a sedation score. We were aiming to determine the incidence of recall with current clinical practice, rather than by using a formal scoring system. A future study could examine the effect of using a sedation score on the incidence of recall, and compare that to current clinical practice. Also, the nurses and physicians were not fully blinded to each others' scores. The nurse section and the physician section were on opposite sides of the same sheet of paper. The physician section was completed first. It is possible that the nurses were influenced by the physicians' score, although we noted that the physicians consistently rated themselves lower than the nurse rating.

Also, there was risk of interviewer bias, as the investigators conducted their own telephone follow-up, and the treating nurses who asked questions after a procedure were unblinded and not independent.

\section{CONCLUSIONS}

Recall following PS in the ED is uncommon, but is important for patient satisfaction. Although clinical staff satisfaction with sedation is adversely affected by the presence of a grimace or groan during the procedure, this has no effect on patient satisfaction, the likelihood of procedural success, or the presence of any recall. There is very high patient satisfaction with PS in the ED. Use of propofol may be positively correlated with physician, nurse and patient satisfaction. Midazolam use may be correlated with a decreased likelihood of delayed recall. Further studies with predetermined drug regimens are required to further define these issues.

\section{Authors' affiliations}

Alex Swann, Department of Anaesthesia, Royal Perth Hospital, Perth, Western Australia, Australia

Jeffrey Williams, Emergency Department, Royal Perth Hospital, Perth,

Western Australia, Australia

Daniel M Fatovich, University of Western Australia, Perth, Western

Australia, Australia

Competing interests: None declared.

Presented at: Australasian College for Emergency Medicine, 2006 Winter Symposium, Townsville, 29 June to 2 July 2006

\section{REFERENCES}

1 Miner JR, Biros MH, Heegaard W, et al. Bispectral electroencephalographic analysis of patients undergoing procedural sedation in the emergency department. Acad Emerg Med 2003:10:638-43.

2 Swanson ER, Seaberg DC, Mathias S. The use of propofol for sedation in the emergency department. Acad Emerg Med 1996;3:234-8.

3 Lerman B, Yoshida D, Levitt MA. A prospective evaluation of the safety and efficacy of methohexital in the emergency department. Am J Emerg Med 1996;14:351-4.

4 Taylor DM, O'Brien D, Ritchie P, et al. Propofol versus midazolam/fentanyl for reduction of anterior shoulder dislocation. Acad Emerg Med 2005;12:13-19.

5 Wright SW, Chudnofsky CR, Dronen SC, Kothari R, Birrer P, Blanton DM, et al Comparison of midazolam and diazepam for conscious sedation in the emergency department. Ann Emerg Med 1993;22:201-5.

6 Miner JR, Bachman A, Kosman L, et al. Assessment of the onset and persistence of amnesia during procedural sedation with propofol. Acad Emerg Med 2005; 12:491-6.

7 Myles PS, Leslie K, McNeil J, et al. Bispectral index monitoring to prevent awareness during anaesthesia: the B-Aware randomised controlled trial. Lancet 2004;363:1757-63.

8 Ghoneim M. Awareness during anaesthesia. Oxford: Butterworth Heinemann, 2001.

9 Ruth WJ, Burton JH, Bock AJ. Intravenous etomidate for procedural sedation in emergency department patients. Acad Emerg Med 2001;8:13-18.

10 Bulach R, Myles PS, Russnak M. Double-blind randomized controlled trial to determine extent of amnesia with midazolam given immediately before general anaesthesia. Br J Anaesth 2005:94:300-5.

11 Dunn MJG, Mitchell R, Souza CD, et al. Evaluation of propofol and remifentanil for intravenous sedation for reducing shoulder dislocations in the emergency department. Emerg Med J 2006;23:57-8.

12 Gill M, Green SM, Krauss B. A study of the Bispectral Index Monitor during procedural sedation and analgesia in the emergency department. Ann Emerg Med 2003;41:234-41.

13 Fatovich DM, Gope M, Paech MJ. A pilot trial of BIS monitoring for procedural sedation in the emergency department. Emerg Med Australas 2004;16:103-7. 